\title{
The Uniformization Problem for Complex Manifolds
}

\author{
by \\ Yum-Tong SIU*
}

The classical uniformization theorem of Riemann surfaces says that a simply connected Riemann surface is conformally equivalent to the Riemann sphere, the complex line, or the open unit 1-disc. As is well-known, this beautiful fact fails in higher dimensions. The set of complex structures on a nice contractible higher-dimensional complex manifold is huge and cannot be classified in such a simple manner. Therefore, in order to find a simple analog of the above uniformization theorem, one must impose more restrictions on the manifold. In this regard, one notices that the uniformization theorem can also be understood in differential geometry in the following manner.

(i) Every compact surface with positive curvature is conformally equivalent to the Riemann sphere.

(ii) Every noncompact complete surface with positive curvature is conformally equivalent to the complex line.

(iii) Every simply connected complete surface with curvature bounded from above by a negative constant is conformally equivalent to the open unit 1-disc.

A natural higher-dimensional analog of these three facts would be that in all cases we replace surfaces by Kähler manifolds and in Case (i) the Riemann sphere by a complex projective space, in Case (ii) the complex line by a complex Euclidean space, and in Case (iii) the open unit 1-disc by a bounded domain in a complex Euclidean space. The higher-dimensional analog of Case (i) is known as Frankel's conjecture. Andreotti-Frankel proved that Frankel's conjecture is true when the Kähler manifold has complex dimension two [2]. Kobayashi-Ochiai and Iitaka

Received September 7, 1976.

* Department of Mathematics, Yale University, New Haven, Connecticut 06520, U.S.A. 
had made definite progress when the Kähler manifold has complex dimension three. They proved that the manifold must be rational [6]. Very little is known about the rest of Frankel's conjecture and the higherdimensional analogs of Cases (ii) and (iii).

Here we give the following result on the higher-dimensional analog of Case (ii) of the uniformization problem. This result is the joint work of myself and S.-T.Yau. It was announced in [8]. Full details will appear in [9].

Main Theorem. If $M$ is a simply connected, complete, Kähler manifold of complex dimension $n$ with

$$
0 \geqq \text { sectional curvature } \geqq-A r^{-2-p}
$$

where $A$ and $p$ are positive numbers and $r$ is the distance from a fixed point of $M$, then $M$ is biholomorphic to $\mathbb{C}^{n}$.

This result was recently conjectured by Greene-Wu [4]. Prior to making this conjecture they had already observed [3] that under the hypothesis of our Main Theorem there exist no nonconstant bounded harmonic functions on $M$. They based their analysis on an application of Moser's Harnack inequality [7]. They also observed that in our Main Theorem one cannot allow $p \leqq 0$. The metric $\left(1-|z|^{2}\right)^{3} d z \otimes d \bar{z}$ on the open unit 1-disc gives the required counter-example.

Our method is based on the $L^{2}$ estimates of $\bar{\partial}$ of Andreotti-Vesentini [1] and Hörmander [5]. We construct holomorphic functions on $M$ similar to the linear functions on $\boldsymbol{C}^{n}$ and use them to map $M$ into $\boldsymbol{C}^{n}$. In the first step we only know that the map is nonsingular at one point. Then we use the growth property of these "linear" functions to prove certain uniqueness property to conclude that the map is nonsingular everywhere. This depends on the estimate of the growth of the volume of complex subvarieties of $M$. In order to complete the proof of the Main Theorem we have to prove that the above map is proper. In this step we use the above-mentioned uniqueness property to prove that every holomorphic function which has polynomial growth is actually a polynomial 
of the coordinates of the above map. Then we prove that we can always find a holomorphic function with polynomial growth which grows fast enough at infinity. Putting these two intermediate results together, we show that the above map is proper.

\section{References}

[1] Andreotti, A. and Vesentini, E., Carleman estimates for the Laplace-Beltrami operator on complex manifolds, Publ. Math. I. H. E. S., 25 (1965), 81-130.

[2] Frankel, T., Manifolds with positive curvature, Pacific J. Math., 11 (1968), 157-170.

[3] Greene, R. and Wu, H., Curvature and complex analysis, Bull. Amer. Math. Soc., 77 (1971), 1045-1049.

[4] Greene, R. and Wu, H., Analysis on noncompact Kähler manifolds, Lecture Notes of the 1975 Amer. Math. Soc. Summer Institute.

[5] Hörmander, L., An introduction to complex analysis in several variables, Van Nostrand, Princeton, N. J. 1966.

[6] Titaka, S., On some new birational invariants of algebraic varieties and their application to rationality of certain algebraic varieties of dimension 3, J. Math. Soc. Japan, 24 (1972), 384-396.

[7] Moser, J., On Harnack's theorem for elliptic differential equations, Comm. Pure Appl. Math., 14 (1961), 577-591.

[8] Siu, Y.-T. and Yau, S.-T., On the structure of complete simply connected Kähler manifolds with nonpositive curvature, Proc. Nat. Acad. Sci. U.S.A., 73 (1976), 1008.

[9] Siu, Y.-T. and Yau, S.-T. Complete Kähler manifolds with nonpositive curvature of faster than quadratic decay, Ann. of Math. (to appear). 
